\title{
Postpartum Reversible Cerebral Vasoconstriction Syndrome Presenting with Radiological Plethora
}

\author{
Manisha Sharma ${ }^{1}$ Bindu Menon ${ }^{1}$ Gayatri Manam² \\ ${ }^{1}$ Department of Neurology, Apollo Speciality Hospital, Nellore, \\ Andhra Pradesh, India \\ ${ }^{2}$ Department of Radiology, Apollo Speciality Hospital, Nellore, \\ Andhra Pradesh, India \\ Indian J Radiol Imaging 2021;31:764-767.
}

Address for correspondence Manisha Sharma, DM, Department of Neurology, Apollo Speciality Hospital, Pinakini Nagar, Nellore, Andhra Pradesh 524004, India (e-mail: doc.manishasharma@gmail.com).

Abstract
Keywords
- reversible cerebral
vasoconstriction
syndrome
- postpartum
reversible cerebral
vasoconstriction
syndrome
- postpartum cerebral
angiopathy
- posterior reversible
encephalopathy
syndrome

Reversible cerebral vasoconstriction syndrome (RCVS) is a clinicoradiological syndrome characterized by segmental vasoconstriction of cerebral arteries with resolution in most patients within 1 to 3 months. It has been known to be a benign uniphasic reversible illness but cases of fulminant RCVS with mortality have also been reported. The symptoms can be varied with presentations like transient ischemic attack (TIA), ischemic stroke (IS), intracerebral hemorrhage (ICH), subarachnoid hemorrhage (SAH), and posterior reversible encephalopathy syndrome (PRES). We report a postpartum female with RCVS with radiological features of $\mathrm{ICH}, \mathrm{SAH}$, atypical PRES, and atypical clinical presentation as well. RCVS can be frequently missed unless there is a high index of suspicion. Hence clinicians should be aware of the typical and the atypical presentation of RCVS along with the radiological findings with the potential complications. Early suspicion and detection in the emergency help in recovery and reducing morbidity.

\section{Introduction}

Reversible cerebral vasoconstriction syndrome (RCVS) is a distinct clinical and radiological syndrome characterized by thunderclap headache and segmental vasoconstriction of cerebral arteries that resolves by 3 months. Since the late 19th century, multiple case reports suggested its association with various clinical settings and disorders like postpartum state, migraine headaches, unruptured cerebral aneurysms, and the use of vasoactive medications. It has been known by different terms like migrainous vasospasm or angiitis, benign angiopathy of the central nervous system (CNS), postpartum angiopathy, thunderclap headache with reversible vasospasm, cerebral vasculopathy, drug-induced angiopathy, call-fleming syndrome, until 2007 when Calabrese et al unified a single term and kept all disorders under the term "reversible cerebral vasoconstriction syndrome" and also proposed a specific diagnostic criteria. Though it is a reversible and a monophasic illness, it can have various presentations like transient ischemic attack (TIA), ischemic stroke (IS), intracerebral hemorrhage (ICH), subarachnoid hemorrhage (SAH), and posterior reversible encephalopathy syndrome (PRES) leading to increased morbidity and even mortality. It is challenging to differentiate it from aneurysmal SAH and primary angiitis of the CNS (PACNS). ${ }^{1,2}$
DOI https://doi.org/ 10.1055/s-0041-1735923. ISSN 0971-3026.

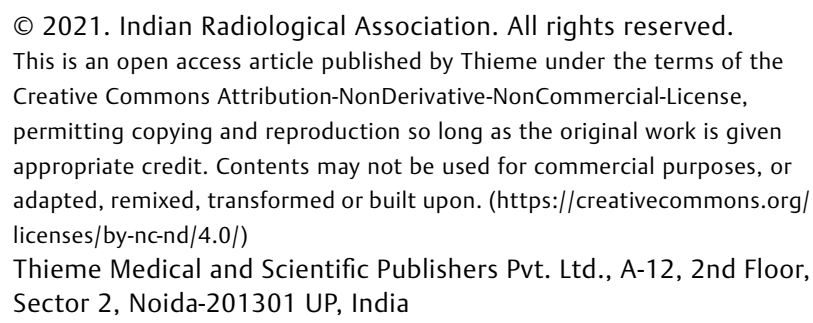


We present a case of postpartum RCVS (PPRCVS), also known as postpartum angiopathy, leading to $\mathrm{ICH}, \mathrm{SAH}$, and atypical PRES with benign course. We present this case to highlight the different clinical presentation and the rare finding of extensive radiological features in a single patient.

\section{Case History}

A 25-year-old pregnant normotensive female, primigravida, with a 9-month period of gestation, visited local hospital for mild labor pains. There was no history of headaches or any medication use during pregnancy. She was conscious, oriented, and was noted to have blood pressure of $180 / 100 \mathrm{~mm}$ $\mathrm{Hg}$. On routine blood investigations, there was leukocytosis (total leukocyte count $25,000 / \mathrm{mm}^{3}$ ), thrombocytopenia (absolute platelet count $44,000 / \mathrm{mm}^{3}$ ), impaired liver enzymes (serum glutamic pyruvic transaminase [SGPT]: $126 \mathrm{mg} \%$ ), hyperbilirubinemia (total bilirubin: $1.8 \mathrm{mg} \%$ ), and urine albumin 2+ suggestive of preeclampsia complicated by HELLP syndrome (hemolysis, elevated liver enzymes, and low platelets). Emergency caesarian section was done. Patient remained asymptomatic for next 24 hours.
On postoperative day 2 , she had one episode of generalized tonic-clonic seizure associated with tongue bite. Patient was managed with antiepileptics, antihypertensives including magnesium sulfate, and intravenous antibiotics. On postoperative day 3, she developed bilateral symmetrical partial ptosis with no fatiguability or diurnal variation. She was further referred to the neurological emergency services of our hospital for further management.

On arrival to emergency, she was conscious and oriented with Glasgow coma scale of 15/15. Blood pressure was 170/ $100 \mathrm{~mm} \mathrm{Hg}$. There was bilateral pitting type pedal edema. She had bilateral symmetrical partial ptosis with no fatiguability. There was right horizontal gaze restriction. Pupils were mid constricted reacting to light. Fundus examination was normal. There was no motor deficit. Patient denied any history of headache. Magnetic resonance imaging (MRI) brain + MR angiogram (MRA) brain + MR venogram brain was done (-Fig. 1) which showed asymmetrical hyperintense signal on T2/fluid-attenuated inversion recovery (FLAIR) in bilateral thalamocapsuloganglionic region and brainstem (pons and midbrain) with no typical diffusion restriction suggestive of vasogenic edema. There were small areas of acute hemorrhage
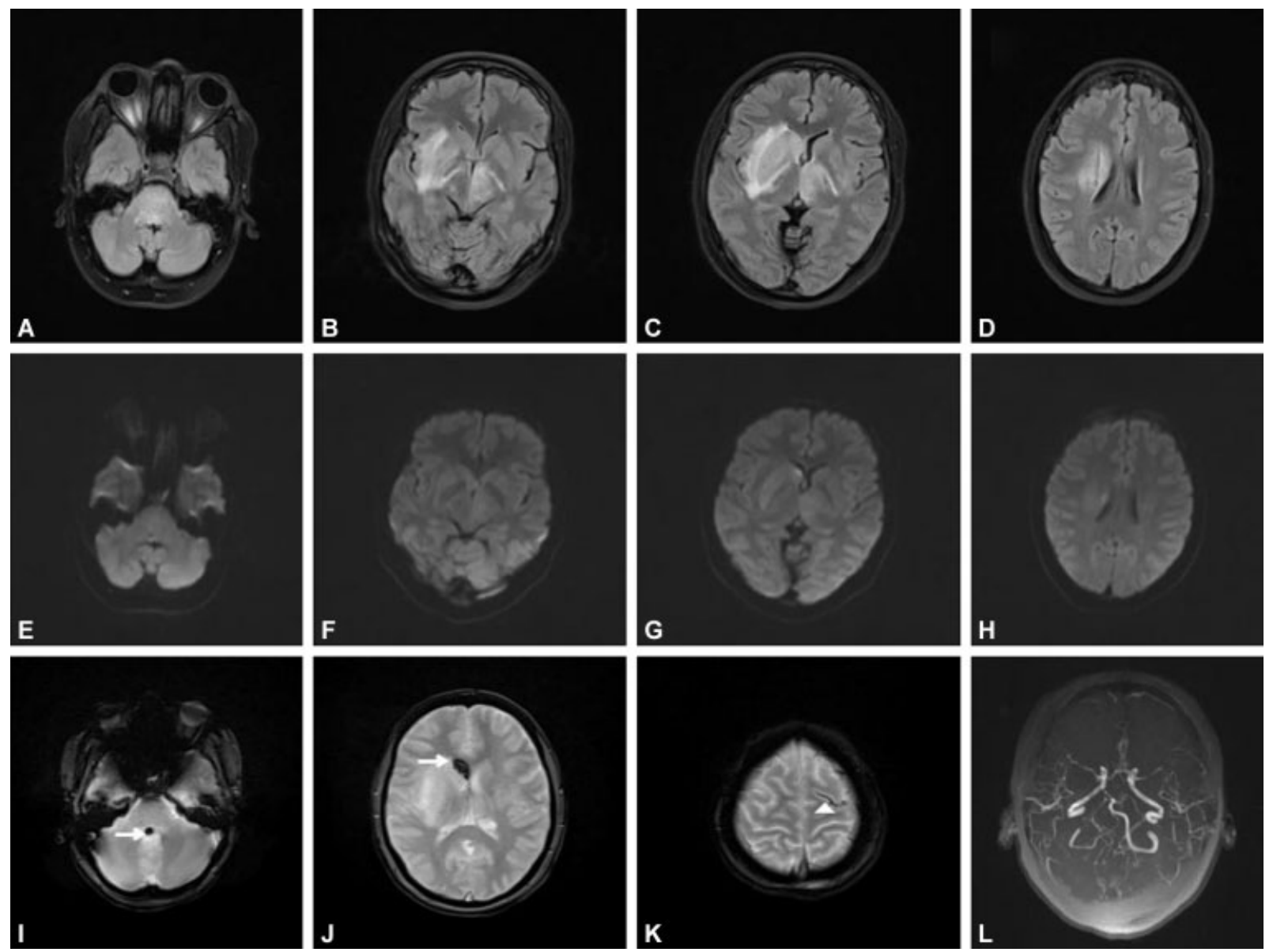

Fig. 1 MRI brain axial FLAIR images (A-D) show asymmetrical (right $>$ left) hyperintense signal in bilateral thalamocapsuloganglionic region and brainstem (pons and midbrain) with no typical diffusion restriction (E-H) suggestive of vasogenic edema. GRE sequences (I-K) show small areas of acute hemorrhage in the posterior pons (I; arrow) and right caudate nucleus (J; arrow). Linear areas of GRE blooming present in left frontal sulci (K; arrow head) suggestive of SAH. MRA brain (L) shows segmental multifocal decreased flow related signal in bilateral middle cerebral arteries (MCAs) and bilateral posterior cerebral arteries (PCAs). FLAIR, fluid-attenuated inversion recovery; MRA, magnetic resonance angiogram; MRI, magnetic resonance imaging; $\mathrm{SAH}$, subarachnoid hemorrhage. 
seen in the posterior pons and right caudate nucleus. Linear areas of GRE (gradient recalled echo) blooming in left frontal sulci suggestive of SAH was present. MRA brain was showing segmental multifocal decreased flow-related signal in bilateral middle cerebral arteries (MCA) and bilateral posterior cerebral arteries (PCA) in both proximal and distal parts. MR Venogram brain was normal. Cerebrospinal fluid (CSF) analysis showed protein, $40 \mathrm{mg} / \mathrm{dL}$; sugar, $55 \mathrm{mg} / \mathrm{dL}$; and 5 cells $/ \mathrm{mm}^{3}$. Based on the radiological findings a diagnosis of postpartum RCVS was made. Our patient fulfilled $4 / 6$ of the proposed diagnostic criteria for RCVS. ${ }^{1}$

Patient was managed with antiepileptic drugs, antihypertensives (injectable magnesium sulfate and oral nifedipine), intravenous steroids (dexamethasone), intravenous antibiotics, and supportive medications with adequate hydration. No seizures recurred during hospital stay. Ptosis and gaze abnormality completely recovered over a period of 1 week. Repeat MRI brain done at 1-week interval was suggestive of partial resolution of vasogenic edema and MRA brain was normal study showing no flow-related signal in bilateral MCAs and PCAs. Patient was discharged at 1 week. On followup as outpatient visit at 1 month, patient was asymptomatic with MRI brain showing near total resolution of PRES changes and normal angiogram (-Fig. 2).

\section{Discussion}

Puerperium has been reported as a risk factor in 7 to $9 \%$ of all RCVS cases. Incidence of PPRCVS is rare as cases often go misdiagnosed. Association of PPRCVS with pregnancy and the puerperium has been proposed to be contributed by female reproductive hormones and pregnancy -related physiological changes in blood volume, blood pressure, and blood vessels leading to alterations in cerebral arterial tone. ${ }^{3}$ Frequent use of vasoactive drugs to reduce postpartum hemorrhage and suppress lactation may be a risk factor for RCVS. ${ }^{4}$ Out of 98 PPRCVS cases studied by Skeik et al, all cases were observed within 0 to 30 days postpartum, with a median occurrence of 5 days. ${ }^{3}$ In our patient, symptoms appeared on second postpartum day.

Recurrent thunderclap headaches have been reported in $94 \%$ of RCVS cases which occurred over a mean period of 1 week. ${ }^{5}$ Our patient did not have headache during the entire course of the illness. Various studies concluded that RCVS can also manifest with unspecific headache, such as a single severe headache episode, a mild, or a progressive headache. Also, $15 \%$ of cases may present without any headache., Patients without history of headache usually present with seizures, focal neurological deficits, confusion, or coma in the
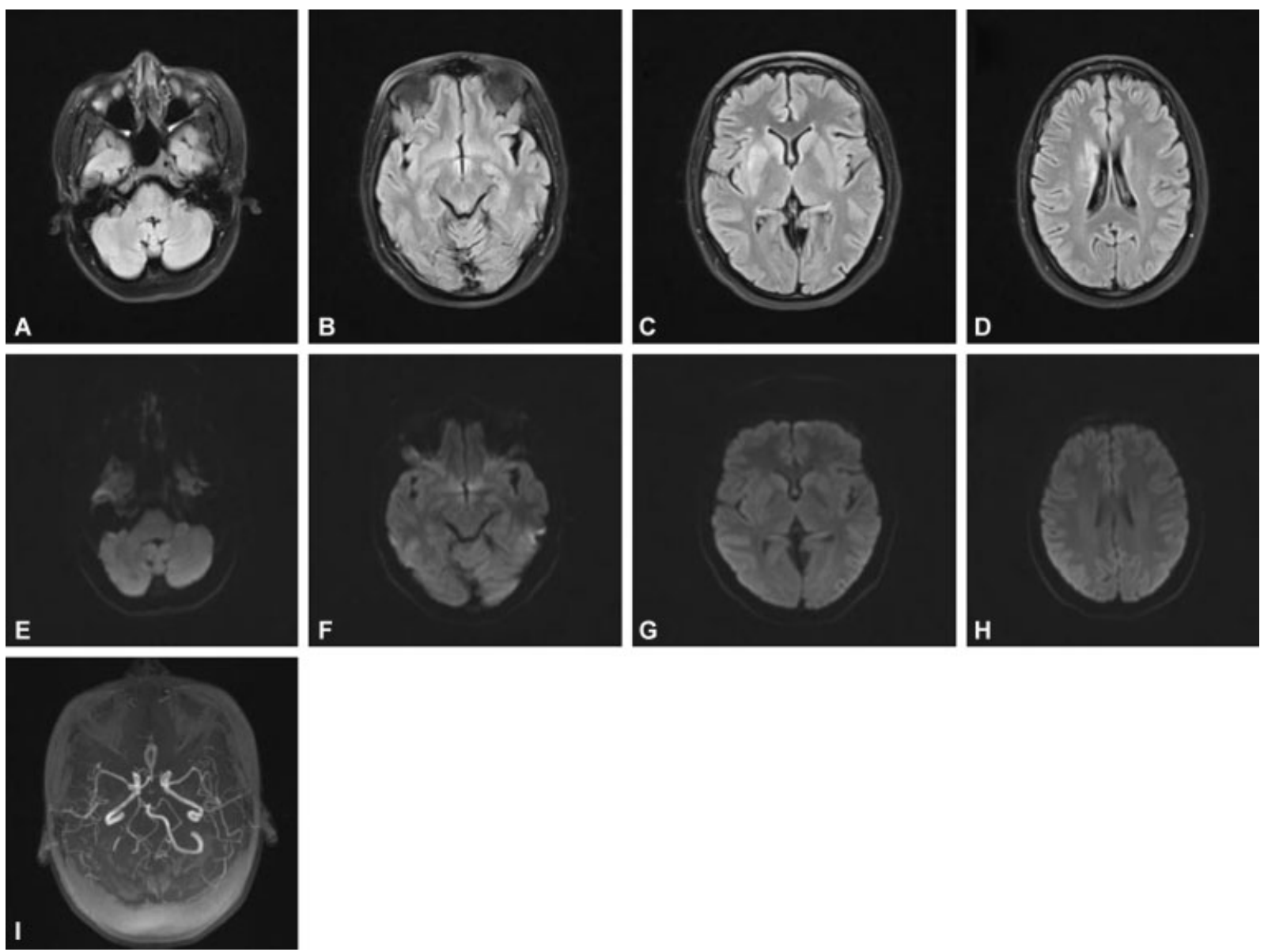

Fig. 2 MRI brain axial FLAIR images (A-D) show hyperintense signal in right thalamocapsuloganglionic region with no diffusion restriction ( $E-H)$ suggestive of resolving edema. MRA brain (I) show normal study with no flow related abnormal signal. FLAIR, fluid-attenuated inversion recovery; MRA, magnetic resonance angiogram; MRI, magnetic resonance imaging. 
setting of stroke or PRES. Headache as an initial manifestation that could be possibly missed in aphasic and comatose patients as well. In such cases, diagnosis relies on radiological demonstration of reversible multifocal intracranial arterial stenosis and the exclusion of other causes. ${ }^{8}$ In our case, absence of headache remained unexplained in absence of aphasia and coma.

Complications in RCVS have been categorized as early and late. Convexity nonaneurysmal SAH (22\%), PRES (9\%), ICH (6\%), and seizures (3\%) occur early within a week as in our case. TIA (16\%) and IS (4\%) occur as late events during the second week. ${ }^{5}$

Convexity SAH are commonly associated with RCVS as in present case. These are nonaneurysmal, usually mild, unilateral, or bilateral, and found in a few sulcal spaces near the convexity. Parenchymal hemorrhages are of variable volume, more frequently single than multiple, and lobar than deep in contrary to present case, where ICH presents at two sites and moreover at deeper areas in caudate and posterior pons. Infarctions occur mainly in arterial watershed regions of the cerebral hemispheres, often between the posterior circulation and the carotid territories. ${ }^{9}$

There is a significant clinical and radiological overlap between RCVS and PRES. Reversible vasogenic edema has been demonstrated in 9 to $38 \%$ of patients with RCVS usually diagnosed within a few days of clinical onset. There is complete reversal of edema usually within 1 month of clinical onset, much earlier than the vasoconstriction. ${ }^{10,11}$ Our patient had atypical radiological presentation with extensive asymmetrical vasogenic edema in brainstem and basal ganglia versus typical symmetrical subcortical parietooccipital involvement. Atypical presentation has been described earlier. Isolated brainstem or basal ganglia involvement without involvement of the cerebral hemispheres may occur in PRES. ${ }^{12}$ In a series of 76 PRES patients by McKinney et al, involvement included the thalamus (30.3\%), cerebellum (34.2\%), brainstem (18.4\%), and basal ganglia (11.8\%) with unilateral involvement seen in $2.6 \%{ }^{13}$

Diagnostic workup is very important in RCVS for demonstrating vasoconstriction to monitor potential complications and to rule out the closest differentials like aneurysmal SAH and PACNS. ${ }^{14}$ Clinically, RCVS has female preponderance and acute onset thunderclap headache is its characteristic, but PACNS has no sex predilection and onset is subacute to chronic with dull aching headache. Abnormal CSF rules out RCVS which is seen in $>95 \%$ cases of PACNS. Radiologically in PACNS, multiple vascular territory infarcts of various ages are present in contrary to watershed infarcts in RCVS along with $\mathrm{SAH}, \mathrm{ICH}$, and reversible brain edema. On neurovascular imaging, in RCVS, there are diffuse areas of multiple stenosis and dilatation in intracranial cerebral arteries which is reversible within days to weeks whereas in PACNS, angiogram may be normal or indistinguishable from RCVS but irreversible. ${ }^{2}$ So acute onset of symptoms, normal CSF analysis, presence of SAH, ICH, and reversible edema on MRI; and reversible angiographic findings favored diagnosis of RCVS and ruled out PACNS.

\section{Conclusion}

The plethora of radiological findings in our patient of $\mathrm{ICH}$, $\mathrm{SAH}$, and atypical PRES would have been difficult to diagnose without the angiographic features of vasoconstriction. Understanding of the radiological features will help us to take appropriate sequences which will help in diagnosis. Early suspicion, detection, and treatment helped in timely clinicoradiological recovery.

\section{Conflicts of Interest}

None declared.

\section{References}

1 Miller TR, Shivashankar R, Mossa-Basha M, Gandhi D. Reversible cerebral vasoconstriction syndrome, part 1: epidemiology, pathogenesis, and clinical course. AJNR Am J Neuroradiol 2015;36(08): 1392-1399

2 Calabrese LH, Dodick DW, Schwedt TJ, Singhal AB. Narrative review: reversible cerebral vasoconstriction syndromes. Ann Intern Med 2007;146(01):34-44

3 Skeik N, Porten BR, Kadkhodayan Y, McDonald W, Lahham F. Postpartum reversible cerebral vasoconstriction syndrome: review and analysis of the current data. Vasc Med 2015;20(03): 256-265

4 Akhter T, Larsson A, Larsson M, Wikström AK, Naessen T. Artery wall layer dimensions during normal pregnancy: a longitudinal study using noninvasive high-frequency ultrasound. Am J Physiol Heart Circ Physiol 2013;304(02):H229-H234

5 Ducros A, Boukobza M, Porcher R, Sarov M, Valade D, Bousser MG. The clinical and radiological spectrum of reversible cerebral vasoconstriction syndrome. A prospective series of 67 patients. Brain 2007;130(pt 12):3091-3101

6 Mawet J, Boukobza M, Franc J, et al. Reversible cerebral vasoconstriction syndrome and cervical artery dissection in 20 patients. Neurology 2013;81(09):821-824

7 Chen SP, Fuh JL, Lirng JF, Wang YF, Wang SJ. Recurrence of reversible cerebral vasoconstriction syndrome: a long-term follow-up study. Neurology 2015;84(15):1552-1558

8 Wolff V, Ducros A. Reversible cerebral vasoconstriction syndrome without typical thunderclap headache. Headache 2016;56(04): 674-687

9 Ducros A. Reversible cerebral vasoconstriction syndrome. Lancet Neurol 2012;11(10):906-917

10 Aracki-Trenkić A, Stojanov D, Trenkić M, et al. Atypical presentation of posterior reversible encephalopathy syndrome: clinical and radiological characteristics in eclamptic patients. Bosn J Basic Med Sci 2016;16(03):180-186

11 Singhal AB, Hajj-Ali RA, Topcuoglu MA, et al. Reversible cerebral vasoconstriction syndromes: analysis of 139 cases. Arch Neurol 2011;68(08):1005-1012

12 McKinney AM, Jagadeesan BD, Truwit CL. Central-variant posterior reversible encephalopathy syndrome: brainstem or basal ganglia involvement lacking cortical or subcortical cerebral edema. AJR Am J Roentgenol 2013;201(03):631-638

13 McKinney AM, Short J, Truwit CL, et al. Posterior reversible encephalopathy syndrome: incidence of atypical regions of involvement and imaging findings. AJR Am J Roentgenol 2007;189 (04):904-912

14 Miller TR, Shivashankar R, Mossa-Basha M, Gandhi D. Reversible cerebral vasoconstriction syndrome, part 2: diagnostic work-up, imaging evaluation, and differential diagnosis. AJNR Am J Neuroradiol 2015;36(09):1580-1588 